\title{
FEEDBACK DESIGN OF DIFFERENTIAL EQUATIONS OF RECONSTRUCTION FOR SECOND-ORDER DISTRIBUTED PARAMETER SYSTEMS
}

\author{
VYACHESLAV I. MAKSIMOV ${ }^{a, b, *}$, BORIS S. MORDUKHOVICH ${ }^{c, d}$ \\ ${ }^{a}$ Institute of Mathematics and Mechanics \\ Ural Branch of the Russian Academy of Sciences, S. Kovalevskaya 16, Yekaterinburg 620990, Russia \\ e-mail: maksimov@imm. uran.ru \\ ${ }^{b}$ Graduate School of Economics and Management \\ Ural Federal University, Yekaterinburg 620002, Russia \\ ${ }^{c}$ Department of Mathematics \\ Wayne State University, Detroit, MI 48202, USA \\ e-mail: boris@math.wayne.edu \\ ${ }^{d}$ Department of Mathematics \\ RUDN University, Moscow 117198, Russia
}

\begin{abstract}
The paper aims at studying a class of second-order partial differential equations subject to uncertainty involving unknown inputs for which no probabilistic information is available. Developing an approach of feedback control with a model, we derive an efficient reconstruction procedure and thereby design differential equations of reconstruction. A characteristic feature of the obtained equations is that their inputs formed by the feedback control principle constructively approximate unknown inputs of the given second-order distributed parameter system.
\end{abstract}

Keywords: second-order partial differential equation, equations of reconstruction.

\section{Introduction}

We consider the following second-order differential equation:

$$
\begin{aligned}
\ddot{y}(t, \eta)-\Delta y(t, \eta)+m y(t, \eta)+\gamma \dot{y}(t, \eta) & \\
= & g(y(t, \eta))+(B v(t))(\eta)+f(t, \eta)
\end{aligned}
$$

for almost every (a.e.) $(t, \eta) \in T \times \Omega$,

with the boundary condition $\left.y(t)\right|_{G}=0$ for a.e. $t \in T$, and the initial conditions.

$$
\begin{aligned}
& y(0)=y_{0} \in V=H_{0}^{1}(\Omega), \\
& \dot{y}(0)=y_{10} \in H=L_{2}(\Omega)
\end{aligned}
$$

Here, $T=[0, \vartheta], 0<\vartheta<+\infty, \Omega$ is an open bounded domain with the Lipschitz boundary $G, \Delta$ is the Laplace

\footnotetext{
*Corresponding author
}

operator, $m=$ const $>0, \gamma=$ const $>0, g(\cdot): \mathbb{R} \rightarrow \mathbb{R}$ is a Lipschitz function with some constant $L, g(0)=0$, $f(\cdot) \in L_{\infty}(T ; H)$ is a given function, and $B$ is a linear continuous operator acting from a Hilbert space $U$ with a norm $|\cdot|_{U}$ and an inner product $(\cdot, \cdot)_{U}$ (the space of disturbances) into the space $H(B \in L(U ; H))$.

Before formulating our main problem, we define a solution of Eqn. (1). Any function $y(\cdot) \in C(T ; V)$ such that

$$
\begin{aligned}
\dot{y}(\cdot) \in W(T ; V)=\{x(\cdot) \in C(T ; H) & : \\
\dot{x}(\cdot) & \left.\in L_{2}\left(T ; V^{*}\right)\right\}
\end{aligned}
$$

and $y(\cdot)$ satisfies

$$
\begin{aligned}
\ddot{y}(t)-\Delta y(t) & +m y(t)+\gamma \dot{y}(t) \\
& =g(y(t))+B v(t)+f(t)
\end{aligned}
$$

in $V^{*}$ for a.e. $t \in T$ 
is called a solution of Eqn. (1) on the interval $T$ and is defined by $y(\cdot)=y\left(\cdot ; y_{10}, y_{0}, u(\cdot)\right)$. Due to Gajewski et al. (1974), for any $v(\cdot) \in L_{2}(T ; U)$, there exists a unique solution of Eqn. (1) on the interval $T$.

A function $v(\cdot)$ (an input) on the right-hand side of Eqn. (1) is unknown. It is only known that this function is an element of the space $L_{2}(T ; U)$. Along with Eqn. (1), there is one more equation of a similar form, namely

$$
\begin{gathered}
\ddot{x}(t)-\Delta x(t)+m x(t)+\gamma \dot{x}(t)=g(x(t)) \\
+B u(t)+f(t) \quad \text { in } V^{*} \text { for a.e. } t \in T
\end{gathered}
$$

with the initial condition

$$
x(0)=x_{0}^{h} \in V, \quad \dot{x}(0)=x_{10}^{h} \in H .
$$

At every time moment $t$, the derivatives of solutions of Eqns. (1) and (3) are measured; i.e., the values $\dot{y}(t)$ and $\dot{x}(t)$ are defined. These measurements can be performed with errors; i.e., instead of functions $\dot{y}(\cdot)$ and $\dot{x}(\cdot)$, we know some functions $\xi^{h}(\cdot) \in L_{\infty}(T ; H)$ and $\psi^{h}(\cdot) \in$ $L_{\infty}(T ; H)$ with the properties

$$
\begin{gathered}
\left|\dot{y}(t)-\xi^{h}(t)\right|_{H} \leq h, \\
\left|\dot{x}(t)-\psi^{h}(t)\right|_{H} \leq h \quad \text { for a.e. } t \in T .
\end{gathered}
$$

In the latter case, we assume that the initial states of Eqns. (1) and (3) satisfy the relations

$$
\left|x_{10}^{h}-y_{10}\right|_{H} \leq h, \quad\left|x_{0}^{h}-y_{0}\right|_{V} \leq h .
$$

Here and below, $h \in(0,1)$ is a value of the measurement error, the symbol $|\cdot|_{H}\left(|\cdot|_{V}\right)$ stands for the norm in the space $H(V)$, and the symbol $(\cdot, \cdot)_{H}$ denotes the scalar product in the space $H$.

In the case where the solutions of Eqns. (1) and (3) are measured with no error (then $x_{0}^{h}=y_{0}$ and $x_{10}^{h}=$ $\left.y_{10}\right)$, it is necessary to specify a family of functions $u^{\alpha}(\cdot)$ (depending on a parameter $\alpha \in(0,1)$ ) with the following properties. First, at every time $t \in T$, the functions $u^{\alpha}(\cdot)$ depend on the derivatives of solutions $\dot{y}(t)$ and $\dot{x}^{\alpha}(t)$, i.e.,

$$
u^{\alpha}(t)=u^{\alpha}\left(\dot{y}(t), \dot{x}^{\alpha}(t)\right),
$$

where $x^{\alpha}(\cdot)=x\left(\cdot ; y_{10}, y_{0}, u^{\alpha}(\cdot)\right)$. Second, the following convergences:

$$
\begin{gathered}
u^{\alpha}(\cdot) \rightarrow u_{*}(\cdot) \quad \text { in } L_{2}(T ; U), \\
x^{\alpha}(\cdot) \rightarrow y(\cdot) \text { in } W(V ; H) \text { as } \alpha \rightarrow 0
\end{gathered}
$$

take place. Here, the symbol $x^{\alpha}(\cdot)=x\left(\cdot ; y_{10}, y_{0}, u^{\alpha}(\cdot)\right)$ denotes the solution of Eqn. (3) with the right-hand side $u(t)=u^{\alpha}(t)$; i.e., $x^{\alpha}(\cdot)$ is the solution of the equation

$$
\begin{aligned}
\ddot{x}(t)-\Delta x(t)+m x(t)+\gamma \dot{x}(t) & \\
=g(x(t))+B u^{\alpha}(t)+f(t) & \\
& \quad \text { in } V^{*} \text { for a.e. } t \in T
\end{aligned}
$$

with the initial condition (4) (with $x_{0}^{h}=y_{0}, x_{10}^{h}=y_{10}$ ),

$$
W(V ; H)=\{w(\cdot) \in C(T ; V): \dot{w}(\cdot) \in C(T ; H)\} .
$$

Further, $u_{*}(\cdot)=u_{*}(\cdot ; y(\cdot))$ means an element of the set $U(y(\cdot))$ of minimal $L_{2}(T ; U)$-norm and $U(y(\cdot))$ is the set of all functions $u(\cdot) \in L_{2}(T ; U)$ generating the solution $y(\cdot)$ of Eqn. (1), i.e.,

$$
\begin{aligned}
U(y(\cdot)) \\
=\left\{u(\cdot) \in L_{2}(T ; U):\right. \\
\quad(\ddot{y}(t)-\Delta y(t)+m y(t)+\gamma \dot{y}(t), z)_{H} \\
\left.=(B u(t)+f(t), z)_{H} \text { for a.e. } t \in T \forall z \in V\right\} .
\end{aligned}
$$

Note that the set $U(y(\cdot))$ 10 is convex and closed in the space $L_{2}(T ; U)$. Therefore, the element $u_{*}(\cdot)$ is unique. In addition, when defining the set $U(y(\cdot))$, we use the solution $y(\cdot)$ of Eqn. (1) generated by some function $v(\cdot) \in L_{2}(T ; U)$. Therefore, the set $U(y(\cdot))$ is non-empty, since it contains at least this function $v(\cdot)$.

Remark 1. Note that one and the same solution of Eqn. (1) can be derived by multiple inputs. In compliance with the approach conventional in the theory of ill-posed problems we reconstruct $u_{*}(\cdot)$.

In the case where solutions of Eqns. (11) and (3) are inaccurately measured (then inequalities (5) and (6) are fulfilled), it is necessary to specify a two-parameter family of functions $u^{\alpha . h}(\cdot)$ (depending on $\alpha \in(0,1)$ and $h \in$ $(0,1))$ with the properties

$$
\begin{aligned}
u^{\alpha, h}(t)=u^{\alpha, h}\left(\xi^{h}(t), \psi^{h}(t)\right), & \\
& \left|\dot{x}^{h}(t)-\psi^{h}(t)\right|_{H} \leq h,
\end{aligned}
$$

and the convergences

$$
\begin{array}{r}
u^{h}(\cdot)=u^{\alpha(h), h}(\cdot) \rightarrow u_{*}(\cdot) \quad \text { in } L_{2}(T ; U), \\
x^{h}(\cdot) \rightarrow y(\cdot) \quad \text { in } W(V ; H) \text { as } h \rightarrow 0,
\end{array}
$$

for an appropriate concordance of $\alpha=\alpha(h)$ and $h$. Here the symbol $x^{h}(\cdot)=x\left(\cdot ; x_{10}^{h}, x_{0}^{h}, u^{h}(\cdot)\right)$ stands for the solution of Eqn. (3) with the right-hand side $u(t)=$ $u^{\alpha, h}(t)$; i.e., $x^{h}(\cdot)$ is the solution of the equation

$$
\begin{aligned}
\ddot{x}(t)-\Delta x(t)+m x(t)+\gamma \dot{x}(t) & \\
=g(x(t))+B u^{h}(t)+f(t) & \text { in } V^{*} \text { for a.e. } t \in T
\end{aligned}
$$

with the initial condition (4). Equations of the form (9) and (4) with properties (7) and (8) (respectively, equations of the form (13) and (4) with the properties (11) and (12) are called differential equations of reconstruction. Thus, 
the problem under consideration in this paper consists in designing differential equations of reconstruction.

The problem described above belongs to the class of problems of dynamical inversion (Lavrentiev et al., 1980; Schwaller et al., 2013; Banks and Kunisch, 1989; Lasiecka et al., 1999; Mordukhovich and Zhang, 1997; Mordukhovich, 2008; 2011). The methodology of solving this problem suggested below uses an approach described, e.g., by Kryazhimskii and Osipov (1995), Maksimov (2002; 1996; 1995), Maksimov and Pandolfi (2001), Maksimov and Tröltzsch (2006), Kryazhimskii and Maksimov (2010), or Kapustyan and Maksimov (2014). This approach is based on the combination of the principle of feedback control (known in the theory of guaranteed control) with a model (Krasovskii and Subbotin, 1988) and one of the basic methods of ill-posed problems - that of the smoothing functional (Lavrentiev et al., 1980).

Note that problems of dynamical reconstruction were studied by Kryazhimskii and Osipov (1995), Maksimov (2002), Maksimov and Pandolfi (2001), Maksimov and Tröltzsch (2006) or Kryazhimskii and Maksimov (2010). In these papers, systems described by ordinary differential and parabolic equations were considered. For hyperbolic equations and variational inequalities, this approach was developed by Maksimov $(1995 ; 1996)$. In the works of Mordukhovich (2011), Maksimov and Tröltzsch (2006), Kryazhimskii and Maksimov (2010) or Maksimov (1995; 1996), the case where an input is subject to instantaneous constraints $u(t) \in P$, with $P$ being a convex, closed and bounded set from a uniformly convex Banach space, was considered. In the present paper, continuing a series of works (Maksimov, 2002; Maksimov and Pandolfi, 2001), we consider the case where such a constraint is absent. Let us emphasize that the developed approach to the study of dynamic systems in uncertainty conditions is significantly different from the well-known stochastic approach to deal with problems under uncertainties, which in fact has not been largely developed for distributed parameter systems. In our case we do not assume the availability of any probabilistic information on perturbations. The absence of such information is quite realistic in many practical problems, in particular, those governed by PDE systems (see, e.g., Mordukhovich, 2008; 2011).

\section{Equations of reconstruction: The case of precise measurement of solutions}

First, we consider the case where the derivatives of the solutions $y(\cdot)$ and $x(\cdot)$ are measured without any error. Namely, we assume that, at every time $t \in T$, elements $\dot{x}(t) \in H$ and $\dot{y}(t) \in H$ are known. Let the function $u^{\alpha}(\cdot)$ on the right-hand side of Eqn. (9) be defined by the formula

$$
u^{\alpha}(t)=\alpha^{-1} B^{*}\left(\dot{y}(t)-\dot{x}^{\alpha}(t)\right),
$$

where $B^{*}$ is the conjugate operator. Then Eqn. (9) has the form

$$
\begin{array}{r}
\ddot{x}^{\alpha}(t)-\Delta x^{\alpha}(t)+m x^{\alpha}(t)+\gamma \dot{x}^{\alpha}(t) \\
=g\left(x^{\alpha}(t)\right)+\alpha^{-1} B B^{*}\left(\dot{y}(t)-\dot{x}^{\alpha}(t)\right)+f(t) \\
\text { in } V^{*} \text { for a.e. } t \in T .
\end{array}
$$

Theorem 1. Let $\gamma>L \vartheta$. Then the convergences (7) and (8) take place.

The assertion of Theorem 1 follows from Theorem 1.2.1 of Maksimov (2002, p. 23) and Lemma2 given below. To prove this lemma, the following statement is necessary. This result can be treated as a variant of the classical Gronwall lemma, while being different from the usual formulations of the latter (see, e.g., Warga, 1972).

Lemma 1. (Barbashin, 1970) Let $\phi(\cdot)$ and $F(\cdot)$ be nonnegative and integrable functions on some interval $t_{0} \leq$ $t \leq t_{0}+a, a>0$. Let $L$ be a positive constant. If the inequality

$$
\phi(t) \leq F(t)+L \int_{t_{0}}^{t} \phi(s) \mathrm{d} s, \quad t_{0} \leq t \leq t_{0}+a,
$$

is valid, then the estimate

$$
\phi(t) \leq F(t)+L \int_{t_{0}}^{t} \exp \{L(t-s)\} F(s) \mathrm{d} s
$$

takes place.

Lemma 2. Let the functions $u^{\alpha}(\cdot)$ on the right-hand side of Eqn. (9) have the following properties:

$$
\begin{gathered}
\sup _{t \in T}\left\{\left|y(t)-x^{\alpha}(t)\right|_{V}^{2}+\left|\dot{y}(t)-\dot{x}^{\alpha}(t)\right|_{H}^{2}\right\} \leq C_{0} \alpha, \\
\left|u^{\alpha}(\cdot)\right|_{L_{2}(T ; U)} \leq\left|u_{*}(\cdot)\right|_{L_{2}(T ; U)} .
\end{gathered}
$$

Then the convergences (7) and (8) take place.

Here $C_{0}$ is some constant independent of $t$ and $\alpha$. In turn, the inequalities (16) and (17) follow from the following result:

Lemma 3. Let the conditions of Theorem 1 be fulfilled. Then the function $u^{\alpha}(\cdot)$ of the form (14) satisfies the conditions of Lemma 2 
Proof. Let $\mu_{\alpha}(t)=x^{\alpha}(t)-y(t)$. By (15), we conclude that $\mu_{\alpha}(t)$ is a solution of the equation

$$
\begin{aligned}
& \ddot{\mu}_{\alpha}(t)-\Delta \mu_{\alpha}(t)+m \mu_{\alpha}(t)+\gamma \dot{\mu}_{\alpha}(t) \\
& =g\left(x^{\alpha}(t)\right)-g(y(t))+\left(B u^{\alpha}\right)(t)-\left(B u_{*}\right)(t), \quad t \in T
\end{aligned}
$$

with the initial conditions

$$
\mu_{\alpha}(0)=0, \quad \dot{\mu}_{\alpha}(0)=0 .
$$

Introduce the Lyapunov function

$$
\begin{aligned}
V(t)= & \left|\dot{\mu}_{\alpha}(t)\right|_{H}^{2}+\left|\mu_{\alpha}(t)\right|_{V}^{2}+m\left|\mu_{\alpha}(t)\right|_{H}^{2} \\
& +2 \gamma \int_{0}^{t}\left|\dot{\mu}_{\alpha}(\tau)\right|_{H}^{2} \mathrm{~d} \tau \\
& +\alpha \int_{0}^{t}\left|u^{\alpha}(\tau)\right|_{U}^{2} \mathrm{~d} \tau, \quad t \in T .
\end{aligned}
$$

Taking (19) into account, we deduce that

$$
\begin{aligned}
& \dot{V}(t)-\alpha\left|u_{*}(t)\right|_{U}^{2} \\
& =2\left(g\left(x^{\alpha}(t)\right)-g(y(t)), \dot{\mu}_{\alpha}(t)\right)_{H} \\
& \quad+2\left(B\left(u^{\alpha}(t)-u_{*}(t)\right), \dot{\mu}_{\alpha}(t)\right)_{H} \\
& \quad+\alpha\left|u^{\alpha}(t)\right|_{U}^{2}-\alpha\left|u_{*}(t)\right|_{U}^{2} \quad \text { for a.e. } t \in T .
\end{aligned}
$$

Note that (see (14)) for a.e. $t \in T$

$$
\begin{aligned}
u^{\alpha}(t)= & \arg \min \left\{\alpha|v|_{U}^{2}\right. \\
& \left.-2\left(B^{*}\left(\dot{y}(t)-\dot{x}^{\alpha}(t)\right), v\right)_{U}: v \in U\right\} .
\end{aligned}
$$

Using (21), we see that

$$
\begin{aligned}
\dot{V}(t) & -\alpha\left|u_{*}(t)\right|_{U}^{2} \\
& \leq 2\left(g\left(x^{\alpha}(t)\right)-g(y(t)), \dot{\mu}_{\alpha}(t)\right)_{H} \\
& \leq 2 L\left|\mu_{\alpha}(t)\right|_{H}\left|\dot{\mu}_{\alpha}(t)\right|_{H} \\
& \leq\left|\dot{\mu}_{\alpha}(t)\right|_{H}^{2}+L^{2}\left|\mu_{\alpha}(t)\right|_{H}^{2} \leq c V(t),
\end{aligned}
$$

where $c=\max \{1, m\} \max \left\{1, L^{2}\right\}$. In addition,

$$
V(0)=0 \text {. }
$$

In this case, using Lemma 1 the inequality

$$
\int_{0}^{t} e^{c(t-\tau)} \mathrm{d} \tau \leq c^{-1} e^{c t}
$$

(22) and (23), we get

$$
\begin{aligned}
V(t) \leq & \alpha \int_{0}^{t}\left|u_{*}(\tau)\right|_{U}^{2} \mathrm{~d} \tau \\
& +c \alpha \int_{0}^{t}\left\{e^{c(t-\tau)} \int_{0}^{\tau}\left|u^{*}(p)\right|_{U}^{2} \mathrm{~d} p\right\} \mathrm{d} \tau \\
\leq & \alpha\left(1+e^{c \vartheta}\right) \int_{0}^{\vartheta}\left|u_{*}(\tau)\right|_{U}^{2} \mathrm{~d} \tau \leq C_{0} \alpha .
\end{aligned}
$$

The inequality (16) is proved. Let us verify the inequality (17). It is easily seen that the inequality

$$
\begin{aligned}
& V(t)-\alpha \int_{0}^{t}\left|u_{*}(\tau)\right|_{U}^{2} \mathrm{~d} \tau \\
& \quad \leq V(0)+2 L \vartheta \int_{0}^{t}\left|\dot{\mu}_{\alpha}(\tau)\right|_{H}^{2} \mathrm{~d} \tau
\end{aligned}
$$

is true. In this case, from (25) it follows that

$$
\begin{aligned}
& \left|\mu_{\alpha}(t)\right|_{V}^{2}+\left|\dot{\mu}_{\alpha}(t)\right|_{H}^{2}+m\left|\mu_{\alpha}(t)\right|_{H}^{2} \\
& \quad+\alpha \int_{0}^{t}\left\{\left|u^{\alpha}(\tau)\right|_{U}^{2}-\left|u_{*}(\tau)\right|_{U}^{2}\right\} \mathrm{d} \tau \leq V(0) .
\end{aligned}
$$

By virtue of (23) and (26), we obtain (17). The proof is complete.

From Theorem 1 it follows that Eqn. (15) is the differential equation of reconstruction in the case of precise measurements of the solution.

\section{Equations of reconstruction: The case of inaccurate measurement of solutions}

Consider the case where the derivatives of the solution $y(\cdot)$ of Eqn. (1) and the solution $x(\cdot)$ of Eqn. (3) are inaccurately measured. Namely, we assume that, at every time $t \in T$, some elements $\xi^{h}(t)$ and $\psi^{h}(t)$ satisfying (5) are known. Let the function $u^{h}(\cdot)$ in (13) be defined by

$$
u^{h}(t)=\alpha^{-1} B^{*}\left(\xi^{h}(t)-\psi^{h}(t)\right) .
$$

In this case, Eqn. (13) takes the form

$$
\begin{array}{r}
\ddot{x}(t)-\Delta x(t)+m x(t)+\gamma \dot{x}(t) \\
=g(x(t))+\alpha^{-1} B B^{*}\left(\xi^{h}(t)-\psi^{h}(t)\right)+f(t) \\
\text { in } V^{*} \text { for a.e. } t \in T .
\end{array}
$$

Let, as above, $u_{*}(\cdot)=u_{*}(\cdot ; y(\cdot))$ be the element of the set $U(y(\cdot))$ of minimal $L_{2}(T ; U)$-norm. 
Theorem 2. Let $\alpha=\alpha(h) \in(0,1)$ for $h \in(0,1)$. Let also $\gamma>L \vartheta$ and $h \alpha^{-2}(h) \leq C=$ const $>0$ for $h \in$ $(0,1)$. Then the convergences (11) and (12) take place.

The assertion of Theorem 2 follows from the results below.

Lemma 4. Let the conditions of Theorem 2 be fulfilled. Then there exists $h_{*} \in(0,1)$ such that the inequalities

$$
\begin{aligned}
& \sup _{t \in T}\left\{\left|y(t)-x^{h}(t)\right|_{V}^{2}+\mid \dot{y}(t)\right.\left.-\left.\dot{x}^{h}(t)\right|_{H} ^{2}\right\} \\
& \leq C_{1}(\alpha(h)+h), \\
&\left|u^{h}(\cdot)\right|_{L_{2}(T ; U)}^{2} \leq r_{1}(h)\left|u_{*}(\cdot)\right|_{L_{2}(T ; U)}^{2}+r_{2}(h)
\end{aligned}
$$

are fulfilled for $h \in\left(0, h_{*}\right)$. Here

$$
r_{1}(h) \rightarrow 1, \quad r_{2}(h) \rightarrow 0 \quad \text { as } h \rightarrow 0 ;
$$

$C_{1}$ is some constant independent of $t, \alpha$, and $h$.

Proof. By (27) and (5), the inequality

$$
\left|u^{h}(t)\right|_{U}^{2} \leq 2 b^{2} \alpha^{-2}\left(h^{2}+\left|\dot{\mu}_{h}(t)\right|_{H}^{2}\right), \quad t \in T,
$$

is valid. Here $\alpha=\alpha(h), \mu_{h}(t)=x^{h}(t)-y(t)$, and $b=$ $\left|B^{*}\right|_{L(H ; U)}$ is the norm of the linear continuous operator $B^{*} \in L(H ; U)$. In this case, for $t \in T$, we have

$$
\int_{0}^{t}\left|u^{h}(\tau)\right|_{U}^{2} \mathrm{~d} \tau \leq 2 b^{2} \alpha^{-2} \int_{0}^{t}\left|\dot{\mu}_{h}(\tau)\right|_{H}^{2} \mathrm{~d} \tau+c_{1} h^{2} \alpha^{-2} .
$$

It is easily seen that

$$
\begin{aligned}
(B & \left.\left(u^{h}(t)-u_{*}(t)\right), \dot{\mu}_{h}(t)\right)_{H} \\
\leq & \left(B\left(u^{h}(t)-u_{*}(t)\right), \psi^{h}(t)-\xi^{h}(t)\right)_{H} \\
\quad & +c_{2} h\left\{\left|u_{*}(t)\right|_{U}+\left|u^{h}(t)\right|_{U}\right\} \quad \text { for a.e. } t \in T .
\end{aligned}
$$

From (1) and (13), it follows that the function $\mu_{h}(t)$ is a solution of the equation

$$
\begin{aligned}
\ddot{\mu}_{h}(t)-\Delta \mu_{h}(t)+m \mu_{h}(t) & +\gamma \dot{\mu}_{h}(t) \\
=g\left(x^{h}(t)\right)-g(y(t))+ & B\left(u^{h}(t)-u_{*}(t)\right) \\
& \text { in } V^{*} \text { for a.e. } t \in T
\end{aligned}
$$

with the initial conditions

$$
\mu_{h}(0)=y_{0}-x_{0}^{h}, \quad \dot{\mu}_{h}(0)=y_{10}-x_{10}^{h} .
$$

Introduce the Lyapunov function (see (19))

$$
\begin{aligned}
V(t)= & \left|\dot{\mu}_{h}(t)\right|_{H}^{2}+\left|\mu_{h}(t)\right|_{V}^{2} \\
& +m\left|\mu_{h}(t)\right|_{H}^{2}+2 \gamma \int_{0}^{t}\left|\dot{\mu}_{h}(\tau)\right|_{H}^{2} \mathrm{~d} \tau \\
& +\alpha \int_{0}^{t}\left|u^{h}(\tau)\right|_{U}^{2} \mathrm{~d} \tau, \quad t \in T .
\end{aligned}
$$

By virtue of (34), we conclude that for a.e. $t \in T$

$$
\begin{aligned}
& \dot{V}(t)-\alpha\left|u_{*}(t)\right|_{U}^{2} \\
& =2\left(g\left(x^{h}(t)\right)-g(y(t)), \dot{\mu}_{h}(t)\right)_{H} \\
& \quad+2\left(B\left(u^{h}(t)-u_{*}(t), \dot{\mu}_{h}(t)\right)\right)_{H} \\
& \quad+\alpha\left|u^{h}(t)\right|_{U}^{2}-\alpha\left|u_{*}(t)\right|_{U}^{2} .
\end{aligned}
$$

Note that the control $u^{h}(t)$ of the form (27) is defined by the rule

$$
\begin{aligned}
u^{h}(t)= & \arg \min \left\{\alpha|v|_{U}^{2}\right. \\
& \left.-2\left(B^{*}\left(\xi^{h}(t)-\psi^{h}(t)\right), v\right)_{U}: v \in U\right\} .
\end{aligned}
$$

From (35), (32) and (36), we deduce that

$$
\begin{aligned}
\dot{V}(t)-\alpha\left|u_{*}(t)\right|_{H}^{2} \leq & c V(t)+2 c_{2} h\left\{\left|u_{*}(t)\right|_{U}\right. \\
& \left.+\left|u^{h}(t)\right|_{U}\right\} \quad \text { for a.e. } t \in T .
\end{aligned}
$$

Using the inclusion $u_{*}(\cdot) \in L_{2}(T ; U)$, we have

$$
\int_{0}^{\vartheta}\left|u_{*}(\tau)\right|_{U} \mathrm{~d} \tau \leq c_{3}
$$

It follows from this inequality, (37), and the inequality

$$
\begin{aligned}
V(0) & =\left|\dot{\mu}_{h}(0)\right|_{H}^{2}+\left|\mu_{h}(0)\right|_{V}^{2}+m\left|\mu_{h}(0)\right|_{H}^{2} \\
& \leq\left(2+m b_{0}^{2}\right) h^{2},
\end{aligned}
$$

by analogy with (24), that

$$
V(t) \leq c_{4}(h+\alpha)+c_{5} h \int_{0}^{t}\left|u^{h}(\tau)\right|_{U}^{2} \mathrm{~d} \tau .
$$

Here $b_{0}>0$ is a constant such that

$$
|z|_{H} \leq b_{0}|z|_{V} \quad \text { for every } z \in V \text {. }
$$

In turn, from (38), by virtue of 31 , we conclude that

$$
\begin{aligned}
V(t) \leq & c_{4}(h+\alpha) \\
& +c_{6} h \alpha^{-2}\left(h^{2}+\int_{0}^{t}\left|\dot{\mu}_{h}(\tau)\right|_{H}^{2} \mathrm{~d} \tau\right) .
\end{aligned}
$$

From (39), it follows that the estimate

$$
\begin{aligned}
\left|\dot{\mu}_{h}(t)\right|_{H}^{2} \leq & c_{4}(h+\alpha) \\
& +c_{6} h \alpha^{-2}\left(h^{2}+\int_{0}^{t}\left|\dot{\mu}_{h}(\tau)\right|_{H}^{2} \mathrm{~d} \tau\right)
\end{aligned}
$$


is valid. Taking into account the Gronwall lemma, we have, for $t \in T$

$$
\begin{aligned}
& \left|\dot{\mu}_{h}(t)\right|_{H}^{2} \\
& \quad \leq\left(c_{4}(h+\alpha)+c_{6} h^{3} \alpha^{-2}\right) \exp c_{6} t h \alpha^{-2} .
\end{aligned}
$$

Due to the condition of the theorem, for $h \in(0,1)$, we get

$$
h \alpha^{-2}(h) \leq C .
$$

Then, using (41) and (42), we have

$$
\left|\dot{\mu}_{h}(t)\right|_{H}^{2} \leq c_{7}(h+\alpha(h)), \quad t \in T .
$$

In this case, (39) and (43) imply the inequality

$$
V(t) \leq c_{8}(h+\alpha), \quad t \in T .
$$

The inequality (29) follows from this estimate. Then taking into account the Lipschitz property of the function $g(\cdot)$, from 26 we obtain the estimate

$$
\begin{aligned}
V(t)- & \alpha \int_{0}^{t}\left|u_{*}(\tau)\right|_{U}^{2} \mathrm{~d} \tau \\
\leq & V(0)+L^{2} h^{2} \vartheta \mu^{-1} \\
& +(2 L \vartheta+\mu) \int_{0}^{t}\left|\dot{\mu}_{h}(\tau)\right|_{H}^{2} \mathrm{~d} \tau+c_{9} h \\
& +c_{10} h \int_{0}^{t}\left|u^{h}(\tau)\right|_{H}^{2} \mathrm{~d} \tau
\end{aligned}
$$

where the number $\mu>0$ is such that

$$
2 \gamma>2 L \vartheta+\mu
$$

In this case, using (44), we obtain

$$
\begin{aligned}
& \left(\alpha-c_{10} h\right) \int_{0}^{t}\left|u^{h}(\tau)\right|_{U}^{2} \mathrm{~d} \tau \\
& \quad \leq \alpha \int_{0}^{t}\left|u_{*}(\tau)\right|_{U}^{2} \mathrm{~d} \tau+c_{11} h, \quad t \in T .
\end{aligned}
$$

The validity of the inequality (30) follows from 450 and the convergence $h \alpha^{-1}(h) \rightarrow 0$ (as $h \rightarrow 0$ ). In this situation,

$$
\begin{aligned}
& r_{1}(h)=\alpha(h)\left\{\alpha(h)-c_{10} h\right\}^{-1}, \\
& r_{2}(h)=c_{11} h\left\{\alpha(h)-c_{10} h\right\}^{-1} .
\end{aligned}
$$

The lemma is proved.
From Theorem 2, it follows that Eqn. (28) is a differential equation of reconstruction in the case of inaccurate measurements of the solution.

Under some additional conditions, one can rewrite the estimate of the convergence rate (see Theorem 3 below). To derive this estimate, we need the following result.

Lemma 5. (Maksimov, 2002, p. 47) Let $u(\cdot) \in$ $L_{\infty}\left(T ; V^{*}\right), v(\cdot) \in W_{V}(T)$,

$$
\left|\int_{0}^{t} u(\tau) \mathrm{d} \tau\right|_{V^{*}} \leq \varepsilon, \quad|v(t)|_{V} \leq K \quad \forall t \in T .
$$

Then for all $t \in T$, the inequality

$$
\left|\int_{0}^{t}\langle u(\tau), v(\tau)\rangle \mathrm{d} \tau\right| \leq \varepsilon(K+\operatorname{var}(T ; v(\cdot)))
$$

is valid.

Here the symbol $\operatorname{var}(T ; v(\cdot))$ means the variation of the function $v(\cdot)$ over the interval $T$, the symbol $W_{V}(T)$ means the set of functions $y(\cdot): T \rightarrow V$ of bounded variation, and the symbol $\langle\cdot, \cdot\rangle$ means the duality between the spaces $V^{*}$ and $V$.

Theorem 3. Let the conditions of Theorem 2 hold. Let also $U=V$, and let $B$ be the operator of canonical embedding of space $V$ into space $H$, and $u_{*}(\cdot) \in W_{V}(T)$. Then the inequality

$$
\begin{aligned}
\mid u_{*}(\cdot)- & \left.u^{h}(\cdot)\right|_{L_{2}(T ; H)} ^{2} \\
& \leq K\left\{h^{1 / 4}+\alpha^{1 / 4}+\left|r_{1}(h)-1\right|+r_{2}(h)\right\}
\end{aligned}
$$

is valid. Here $K$ is some constant independent of $t, \alpha$, and $h$; the symbol $|\cdot|$ means the absolute value of its argument.

Proof. Taking into account equality (33) and multiplying its right-and left-hand sides by $\dot{\mu}_{h}(t)$, then integrating, we see that

$$
\begin{aligned}
& \left|\dot{\mu}_{h}(t)\right|_{H}^{2}+\left|\mu_{h}(t)\right|_{V}^{2}+m\left|\mu_{h}(t)\right|_{H}^{2}+2 \gamma \int_{0}^{t}\left|\dot{\mu}_{h}(\tau)\right|_{H}^{2} \mathrm{~d} \tau \\
& \leq\left|\dot{\mu}_{h}(0)\right|_{H}^{2}+\left|\mu_{h}(0)\right|_{V}^{2}+m\left|\mu_{h}(0)\right|_{H}^{2} \\
& \quad+2 L \int_{0}^{t}\left|\mu_{h}(\tau)\right|_{H}\left|\dot{\mu}_{h}(\tau)\right|_{H} \mathrm{~d} \tau \\
& \quad+K_{1} \int_{0}^{t}\left|u_{*}(\tau)-u^{h}(\tau)\right|_{V}\left|\dot{\mu}_{h}(\tau)\right|_{V^{*}} \mathrm{~d} \tau .
\end{aligned}
$$


By the Cauchy-Bunyakovsky inequality and Lemma 4 (see (29)), the last term on the right-hand side of (46) is estimated from above by the value

$$
K_{2}\left(h^{1 / 2}+\alpha^{1 / 2}\right) .
$$

Using (6), (46), and (47), we conclude that the estimate

$$
\left|\dot{\mu}_{h}(t)\right|_{H}^{2}+\left|\mu_{h}(t)\right|_{V}^{2} \leq K_{3}\left(h^{1 / 2}+\alpha^{1 / 2}\right)
$$

is valid. Note that, for any $t \in T$, the inequality

$$
\begin{aligned}
& \left|\int_{0}^{t} B\left(u_{*}(\tau)-u^{h}(\tau)\right) \mathrm{d} \tau\right|_{V^{*}} \\
& =\sup _{|v|_{V \leq 1}} \mid\left\langle\int _ { 0 } ^ { t } \left\{\ddot{\mu}_{h}(\tau)-\Delta \mu_{h}(\tau)+m \mu_{h}(\tau)\right.\right. \\
& \left.\left.\quad+\gamma \dot{\mu}_{h}(\tau)-g\left(x^{h}(\tau)\right)+g(y(\tau))\right\} \mathrm{d} \tau, v\right\rangle \mid \\
& \leq K_{4}\left\{\left|\dot{\mu}_{h}(t)-\dot{\mu}_{h}(0)\right|_{H}+\left|\mu_{h}(t)-\mu_{h}(0)\right|_{V}\right. \\
& \left.\quad+\int_{0}^{t}\left|\mu_{h}(\tau)\right|_{V} \mathrm{~d} \tau\right\}
\end{aligned}
$$

is fulfilled. Then, by using (48) and (6), we conclude that

$$
\left|\int_{0}^{t} B\left(u_{*}(\tau)-u^{h}(\tau)\right) \mathrm{d} \tau\right|_{V^{*}} \leq K_{5}\left(h^{1 / 4}+\alpha^{1 / 4}\right) .
$$

By virtue of Lemma 5 and the relations (30) and (50), we obtain

$$
\begin{aligned}
\mid u_{*}(\cdot)- & \left.u^{h}(\cdot)\right|_{L_{2}(T ; H)} ^{2} \\
\leq & \left(1+r_{1}(h)\right)\left|u_{*}(\cdot)\right|_{L_{2}(T ; H)}^{2} \\
& -2 \int_{0}^{\vartheta}\left(B u_{*}(\tau), u^{h}(\tau)\right)_{H} \mathrm{~d} \tau+r_{2}(h) \\
= & 2 \int_{0}^{\vartheta}\left(B\left(u_{*}(\tau)-u^{h}(\tau)\right), u_{*}(\tau)\right)_{H} \mathrm{~d} \tau \\
& +\left|r_{1}(h)-1\right| \int_{0}^{\vartheta}\left|B u_{*}(\tau)\right|_{H}^{2} \mathrm{~d} \tau+r_{2}(h) \\
\leq & K\left\{h^{1 / 4}+\alpha^{1 / 4}+\left|r_{1}(h)-1\right|+r_{2}(h) .\right.
\end{aligned}
$$

The proof of the theorem is complete.

\section{Numerical example}

In this section, we present a numerical example. The problem described in Section 3 is solved. It is assumed that the parameters of Eqn. (1) are as follows:

$$
\Omega=[0,1], \quad \vartheta=2, \quad m=2, \quad \gamma=1, \quad U=\mathbb{R},
$$

$$
\begin{gathered}
f(t, \eta)=0, \quad g(x)=\sin x \\
(B v)(\eta)=\omega(\eta) v, \quad \omega(\eta)=1 \quad \text { for a.e. } \eta \in \Omega .
\end{gathered}
$$

As the initial state of (1), we take the functions $y_{0}(\eta)=$ $\eta(1-\eta), y_{10}(\eta)=\eta$ for a.e. $\eta \in \Omega$. The control on the right-hand side of Eqn. (1) is $v(t)=t^{2}$ and the control on the right-hand side of Eqn. (3) is calculated by (27). Equations (1) and (3) are solved by the grid method with the step $\Delta \omega$ in the domain $\Omega$ and the step $\delta$ in the time interval $T$.

The results of computer modelling are presented in Figs. $1-6$ for the following case: $\Delta \omega=1 / 15, \delta=2 / 150$, $\tau_{i}=\tau_{i-1}+\delta, \tau_{0}=0$. In the experiment, we assume $\xi^{h}\left(\tau_{i}, \nu_{j}\right)=\dot{y}\left(\tau_{i}, \nu_{j}\right)+h, \psi^{h}\left(\tau_{i}, \nu_{j}\right)=\dot{x}\left(\tau_{i}, \nu_{j}\right)+h$, where $\nu_{j}=j \Delta \omega, j=0, \ldots, 1 / \Delta \omega$. Figures 1 and 2 correspond to the case of $h=0$, Figs. 3 and 4 to the case of $h=0.01$, and Figs. 5 and 6 to the case $h=0.1$. In Figs. 1, 3, and 5, the solid line represents the section of the function $\dot{y}(t, \eta)$ by the hyperplane $\eta=0.5$; the dashed line-a similar section of the function $\dot{x}(t, \eta)$. By analogy, in Figs. 2, 4 and 6, the solid line corresponds to the control $v(t)$ and the dashed line to the control $u^{h}(t)$. As can be seen from Fig. 1, the corresponding curves actually coincide.

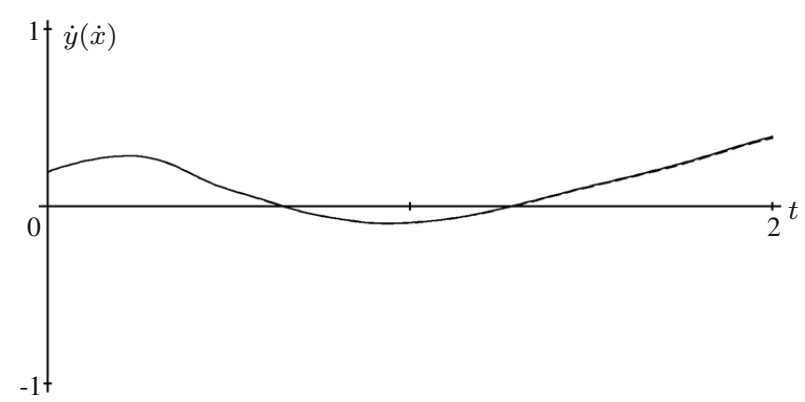

Fig. 1. $h=0$.

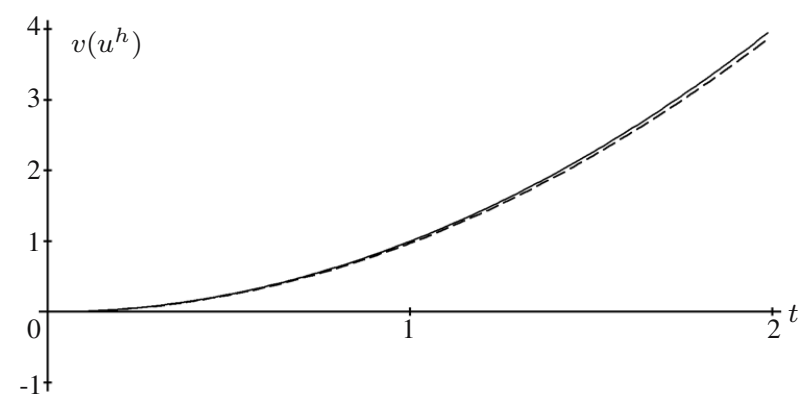

Fig. 2. $h=0$. 


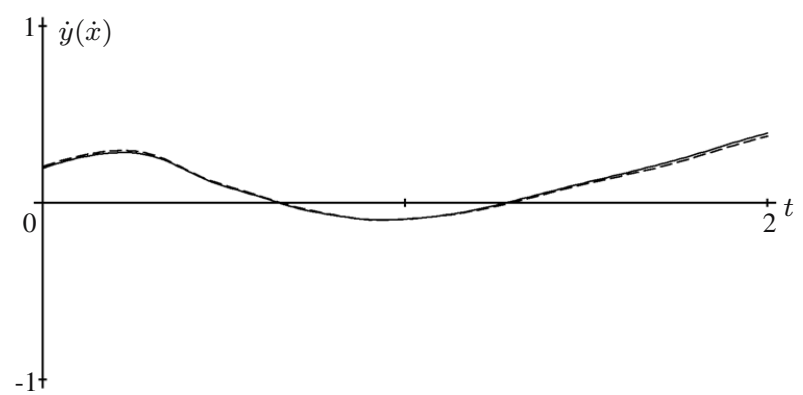

Fig. 3. $h=0.01$

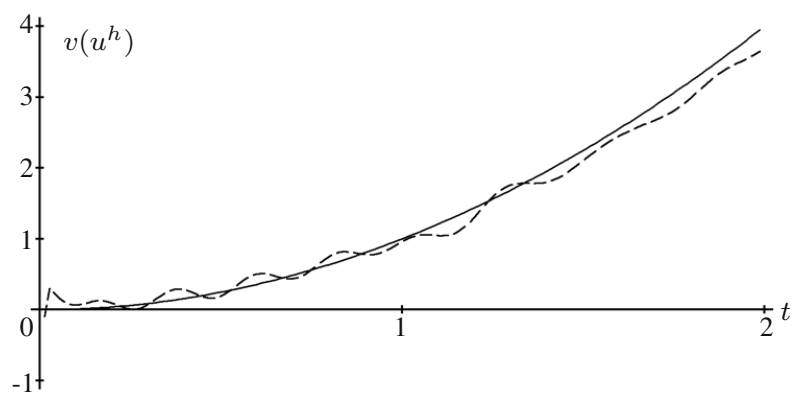

Fig. 4. $h=0.01$.

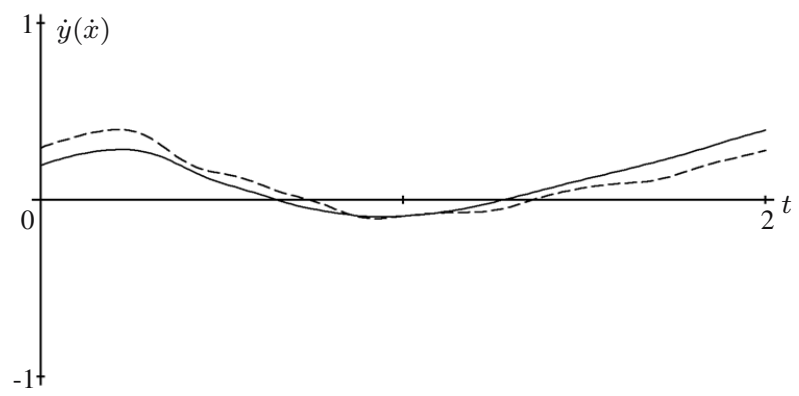

Fig. 5. $h=0.1$.

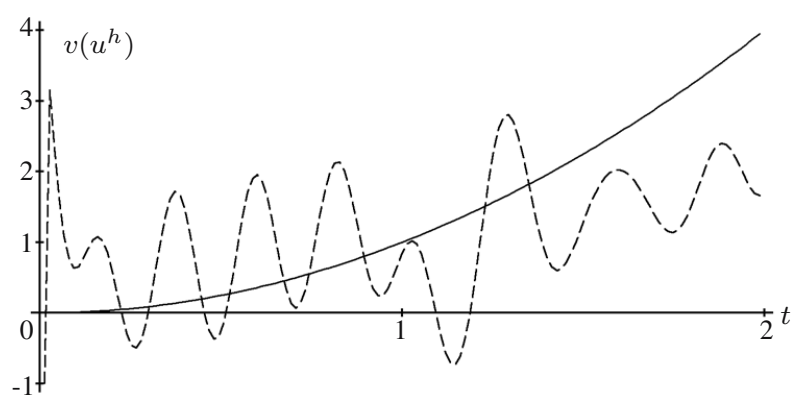

Fig. 6. $h=0.1$.

\section{Conclusions}

For a second-order partial differential equation, an algorithm for constructing a differential equation of reconstruction has been suggested. The problem consists in the design of a reconstruction equation with a feedback control providing the closeness of solutions (and controls) of two equations: the given one (with an unknown control and a solution measured inaccurately) and the sought one (with a control formed by an appropriate way). The performance of the algorithm has been tested on a model example.

\section{Acknowledgment}

The research of the second author was partly supported by the US National Science Foundation under the grant DMS-1512846 and by the US Air Force Office of Scientific Research under the grant 15RT0462.

\section{References}

Banks, H.T. and Kunisch, K. (1980). Estimation Techniques for Distributed Parameter Systems, Birkhäuser, Boston, MA.

Barbashin, E.A. (1970). Introduction to the Theory of Stability, Wolters/Noordhoff, Groningen.

Gajewski, H., Groger, K. and Zacharias, K. (1974). Nichtlineare operatorgleichungen und operator differentialgleichungen, Academic Verlag, Berlin.

Krasovskii, N.N. and Subbotin, A.I. (1988). Game-Theoretial Control Problems, Springer Verlag, New York, NY/Berlin.

Kryazhimskii, A.V. and Osipov, Yu.S. (1995). Inverse Problems for Ordinary Differential Equations: Dynamical Solutions, Gordon and Breach, London.

Kryazhimskii, A.V. and Maksimov, V.I. (2010). Resource-saving infinite-horizon tracking under uncertain input, Applied Mathematics and Computations 217(3): 1135-1140

Kapustyan, V. and Maksimov, V. (2014). On attaining the prescribed quality of a controlled fourth order system, International Journal of Applied Mathematics and Computer Science 24(1): 75-85, DOI: 10.2478/amcs-2014-0006.

Lasiecka, I., Triggiani, R. and Yao, P.-F. (1999). Inverse/observability estimates for second order hyperbolic equations with variable coefficients, Journal of Mathematical Analysis and Applications 235(1): 13-57.

Lavrentiev, M.M., Romanov, V.G. and Shishatskii, S.P. (1980). Ill-Posed Problems of Mathematical Physics and Analysis, Nauka, Novosibirsk, (in Russian).

Maksimov, V.I. (1995). Approximation of an inverse problem for variational inequalities, Differential and Integral Equations 8(8): 2189-2196.

Maksimov, V.I. (1996). Some dynamical inverse problems for hyperbolic systems, Control and Cybernetics 25(3): 465-481. 
Maksimov, V.I. and Pandolfi, L. (2001). The reconstruction of unbounded controls in non-linear dynamical systems, Journal of Applied Mathematics and Mechanics 65(3): 371-380.

Maksimov, V.I. (2002). Dynamics Inverse Problems of Distributed Systems, VSP, Utrecht/Boston, MA.

Maksimov, V.I. and Tröltzsch, F. (2006). Dynamical state and control reconstruction for a phase field model, Dynamics of Continuous, Discrete and Impulsive Systems A: Mathematical Analysis 13(3-4): 419-436.

Mordukhovich, B.S. and Zhang, K. (1997). Minimax control of parabolic equations with Dirichlet boundary conditions and state constraints, Applied Mathematics and Optimization 36(3): 323-360.

Mordukhovich, B.S. (2008). Optimization and feedback design of state-constrained parabolic systems, Pacific Journal of Optimization 4(3): 549-570.

Mordukhovich, B.S. (2011). Optimal control and feedback design of state-constrained parabolic systems in uncertainty conditions, Applicable Analysis 90(6): 1075-1109

Schwaller, B., Ensminger, D., Dresp-Langley, B. and Ragot, J. (2013). State estimation for a class of nonlinear systems, International Journal of Applied Mathematics and Computer Science 23(2): 383-394, DOI: 10.2478/amcs-2013-0029.

Warga, J. (1972). Optimal Control of Differential and Functional Equations, Academic Press, New York, NY.

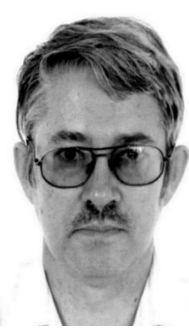

Vyacheslav Maksimov graduated in mathematics and mechanics from the Ural State University, Yekaterinburg, Russia, in 1972. He received his $\mathrm{PhD}$ and DSc degrees in physics and mathematics from the Institute of Mathematics and Mechanics, Ural Branch of the Russian Academy of Sciences, in 1978 and 1992, respectively. Since 1972, he has been with the Institute of Mathematics and Mechanics, Yekaterinburg. Since 1994, he has been a department head at the same institute and a professor at the Chair of Controlled Systems Modeling of the Ural Federal University, Yekaterinburg. He is the author of more than 150 technical publications, including four monographs. His research interests are primarily focused on control theory, distributed parameter systems, and mathematical modeling. Dr. Maksimov is a member of the American Mathematical Society (AMS) and of the IFIP TC7 and WG7.2. He has also been a member of editorial boards of various journals.

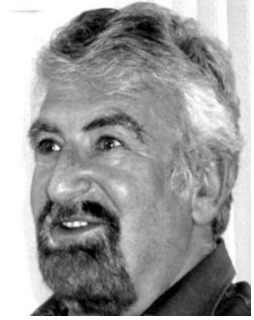

Boris Mordukhovich graduated in applied mathematics from Belarus State University, Minsk, Russia, in 1971, and obtained his $\mathrm{PhD}$ there in 1973. He is currently a distinguished university professor of mathematics at Wayne State University, Detroit, MI, USA. He has around 400 publications, including several monographs and engineering patents. Among his best known mathematical achievements are the introduction and development of powerful constructions of generalized differentiation and their applications to broad classes of problems in variational analysis, optimization, equilibrium, control, economics, engineering, and other fields. Dr. Mordukhovich is a fellow of the American Mathematical Society (AMS) and of the Society of Industrial and Applied Mathematics (SIAM). He is also a recipient of many international awards and honors, including honorary doctorates and professorships from eight universities all over the world, academy memberships, etc. He is listed as a highly cited researcher in mathematics.

Received: 30 November 2016

Revised: 13 March 2017

Re-revised: 25 April 2017

Accepted: 4 May 2017 